\title{
Genetic modification to induce CXCR2 overexpression in mesenchymal stem cells enhances treatment benefits in radiation- induced oral mucositis
}

Zongshan Shen $\mathbb{0}^{1,2}$, Jiancheng Wang ${ }^{2}$, Qiting Huang ${ }^{1}$, Yue Shi ${ }^{2}$, Zhewei Wei ${ }^{3}$, Xiaoran Zhang ${ }^{2}$, Yuan Qiu ${ }^{2}$, Min Zhang ${ }^{2,4}$, Yi Wang ${ }^{2}$, Wei Qin ${ }^{1}$, Shuheng Huang ${ }^{1}$, Yinong Huang ${ }^{2}$, Xin Liư ${ }^{2}$ Kai Xia ${ }^{2,4}$, Xinchun Zhang ${ }^{5}$ and Zhengmei $\operatorname{Lin}^{1}$

\begin{abstract}
Radiation-induced oral mucositis affects patient quality of life and reduces tolerance to cancer therapy. Unfortunately, traditional treatments are insufficient for the treatment of mucositis and might elicit severe side effects. Due to their immunomodulatory and anti-inflammatory properties, the transplantation of mesenchymal stem cells (MSCs) is a potential therapeutic strategy for mucositis. However, systemically infused MSCs rarely reach inflamed sites, impacting their clinical efficacy. Previous studies have demonstrated that chemokine axes play an important role in MSC targeting. By systematically evaluating the expression patterns of chemokines in radiation/chemical-induced oral mucositis, we found that CXCL2 was highly expressed, whereas cultured MSCs negligibly express the CXCL2 receptor CXCR2. Thus, we explored the potential therapeutic benefits of the transplantation of CXCR2-overexpressing MSCS (MSCs ${ }^{\mathrm{CXCR2}}$ ) for mucositis treatment. Indeed, $\mathrm{MSCS}^{\mathrm{CXCR2}}$ exhibited enhanced targeting ability to the inflamed mucosa in radiation/chemical-induced oral mucositis mouse models. Furthermore, we found that MSC ${ }^{\text {CXCR2 }}$ transplantation accelerated ulcer healing by suppressing the production of pro-inflammatory chemokines and radiogenic reactive oxygen species (ROS). Altogether, these findings indicate that CXCR2 overexpression in MSCs accelerates ulcer healing, providing new insights into cell-based therapy for radiation/chemical-induced oral mucositis.
\end{abstract}

\section{Introduction}

Approximately $80-100 \%$ of patients with head and neck cancers who receive radiation treatment develop oral mucositis, which is the most common complication of this treatment $^{1}$. Oral mucositis affects food intake and

Correspondence: Xinchun Zhang (zhxinch@mail.sysu.edu.cn) or Zhengmei Lin (linzhm@mail.sysu.edu.cn)

${ }_{1}^{1}$ Guangdong Provincial Key Laboratory of Stomatology, Department of Operative Dentistry and Endodontics, Guanghua School of Stomatology, Sun Yat-sen University, Guangzhou, China

${ }^{2}$ The Key Laboratory for Stem Cells and Tissue Engineering, Center for Stem Cell Biology and Tissue Engineering, Ministry of Education, Sun Yat-sen

University, Guangzhou, China

Full list of author information is available at the end of the article

Zongshan Shen, Jiancheng Wang, and Qiting Huang contributed equally to this work.

Edited by $Y$ Wang swallowing and speaking ability, ultimately leading to malnutrition, and can lead to life-threatening bacteremia ${ }^{2,3}$, thereby reducing patient tolerance to cancer therapy and patient survival ${ }^{3}$. Previous studies have found that oxidative stress induced by radiation leads to reactive oxygen species (ROS) production, which greatly impacts mucositis because ROS damage DNA, induce cell apoptosis, and increase proinflammatory cytokine release ${ }^{4}$. However, traditional treatments, such as pain management, nutrition support therapy, and antibiotics administration, can alleviate the symptoms of mucositis but are not sufficient for the prevention or treatment of this condition ${ }^{1,4,5}$. Moreover, these treatments elicit severe side effects, such as opportunistic changes were made. The images or other third party material in this article are included in the article's Creative Commons license, unless indicated otherwise in a credit line to the material. If
material is not included in the article's Creative Commons license and your intended use is not permitted by statutory regulation or exceeds the permitted use, you will need to obtain permission directly from the copyright holder. To view a copy of this license, visit http://creativecommons.org/licenses/by/4.0/. 
infections and lipid metabolic disorder. Therefore, it is essential to explore effective treatments with fewer adverse effects.

Because mesenchymal stem cells (MSCs) exhibit beneficial immunomodulatory, anti-oxidative, and antiinflammatory characteristics, MSC therapy has been reported to be effective for patients with a series of inflammatory and radiogenic diseases, including myocardial infarction (MI), spinal cord injury, osteomyelitis, Crohn's disease, and radiogenic skin inflammation ${ }^{6-9}$. These studies indicated that MSC transplantation might represent a promising therapy for radiogenic mucositis. In a clinical setting, MSCs are typically administered through two routes: local transplantation and systemic infusion. Because radiogenic mucositis is distributed in various parts of the human body, local transplantation is not appropriate. Additionally, local implantation has many limitations, such as significant morbidity and disruption of the structure of the local environment ${ }^{10}$. Thus, intravascular administration is much more appropriate. However, the low migratory efficiency of MSCs into the inflamed mucosa limits this approach and reduces its clinical benefits ${ }^{11}$. Therefore, studies aimed at promoting MSC migration toward mucositis sites are vital.

Chemokine axes control the migratory patterns of MSCs to specific sites (i.e., injured sites) ${ }^{12,13}$. Chemokines released from inflammatory tissues might activate adhesion ligands and promote the transendothelial migration or subsequent implantation of MSCs in the surrounding tissues ${ }^{14}$. The targeting of MSCs toward inflamed sites relies on specific chemokine receptors. However, the expression of these receptors in MSCs decreases after in vitro expansion ${ }^{15}$. To enhance their migratory ability, researchers have attempted to overexpress the corresponding receptors in MSCs. In our previous study, CXCR5-overexpressing MSCs exhibited enhanced targeting ability to the inflamed skin in a contact hypersensitivity (CHS) mouse model, in which CXCL13 was notably upregulated. Moreover, these genetically modified MSCs with enhanced targeting ability markedly suppress skin inflammation ${ }^{13}$. Therefore, methods that re-establish the interactions between tissue-specific chemokines and their corresponding receptors on MSCs are promising strategies for enhancing the targeting ability of MSCs and thereby improve the therapeutic benefits of MSC therapy.

Here, overexpression of the chemokine receptor CXCR2 on MSCs improved cell migration to the inflamed mucosa and promoted cell survival in oral radiation/ chemical-induced mucositis (RIM/CIM). Furthermore, CXCR2-overexpressing MSCs (MSCs ${ }^{\text {CXCR2 }}$ ) accelerated ulcer healing, likely by suppressing ROS and proinflammatory chemokine production. Thus, this innovative strategy that enhances the therapeutic benefits shows promise for future clinical applications.

\section{Results}

\section{CXCL2 is upregulated in radiation/chemical-induced oral mucositis}

To systematically investigate the expression of chemokines during the inflammatory phase of RIM/CIM, we evaluated the mRNA expression of chemokines associated with skin and mucosal inflammation, including CCL2, CCL8, CCL17, CCL19, CCL21, CXCL1, CXCL2, CXCL3, CXCL5, CXCL9, CXCL10, and CXCL12 ${ }^{16-19}$. We found that the mRNA levels of various CXCR2 ligands, including CXCL1, CXCL3, CXCL5, and CXCL2, were upregulated. The CXCL2 mRNA levels were markedly upregulated after radiation compared with normal tissues (Fig. 1a). Furthermore, CXCL2 upregulation was confirmed by in situ immunofluorescence staining and western blotting (Fig. 1b, c). Interestingly, the expression of CXCL2 mRNA peaked on day 7 after radiation and then gradually declined (Supplementary Fig. 2A), which was consistent with the clinical symptoms. CIM is another model applied for studying oral mucositis caused by cancer therapy ${ }^{20}$. Similarly, the expression levels of CXCL2 mRNA and protein were substantially increased in CIM (Fig. 1d-f). However, the highest levels of CXCL2 mRNA expression were observed 5 days after chemical induction (Supplementary Fig. 2B). These results indicate that CXCL2 is the dominant chemokine expressed in oral mucositis.

\section{CXCR2-overexpressing MSCs retain the characteristics of human MSCs}

Chemokine receptors play an essential role in MSC targeting to inflamed sites by binding to their corresponding chemokines ${ }^{12,21}$. Therefore, we evaluated the expression of chemokine receptors on MSCs. A transcription quantitative real-time PCR (RT-qPCR) analysis demonstrated that cultured MSCs at passage six expressed very low levels of chemokine receptors, including CXCR2 (Fig. 2a; Supplementary Fig. 2c), which is the receptor of the chemokine that is highly expressed in mucositis, CXCL2.

Subsequently, we overexpressed CXCR2 in MSCs by infecting them with a lentiviral vector encoding CXCR2 to construct $\mathrm{MSCs}{ }^{\mathrm{CXCR} 2}$ (Supplementary Fig. 3A) and potentially enhance the targeting of MSCs to inflamed sites. Moreover, we infected MSCs with a lentiviral vector encoding GFP (referred to as MSCs ${ }^{\text {GFP }}$ ) to allow the tracing of MSCs after transplantation (Supplementary Fig. 3B). Indeed, the CXCR2 expression levels were significantly upregulated 2 days after infection (Fig. 2b). Moreover, this upregulation was further confirmed by western blotting and flow cytometry (Fig. 2c, d). To determine whether CXCR2-overexpressing MSCs retain their identities, we investigated the expression of MSCspecific markers and the multilineage differentiation 


\section{A}
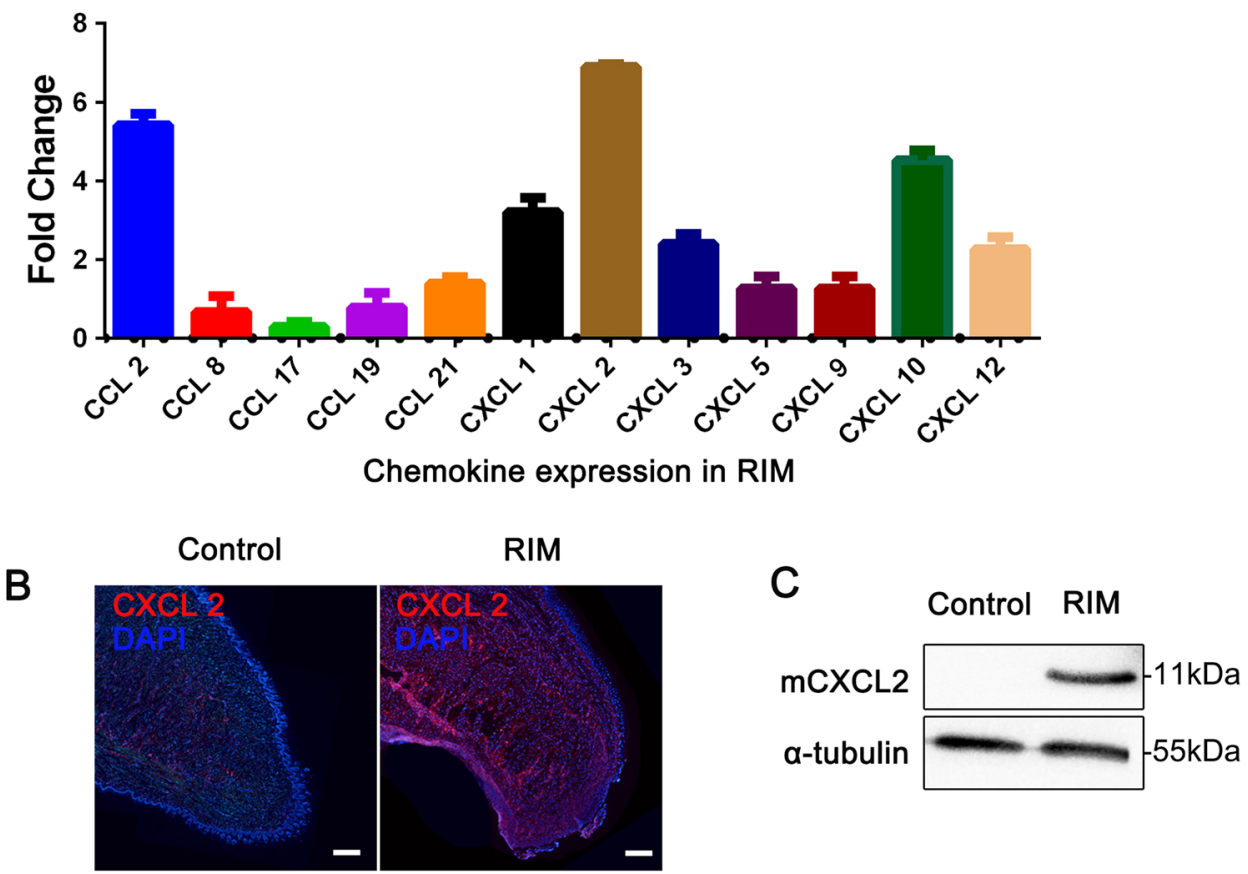

D

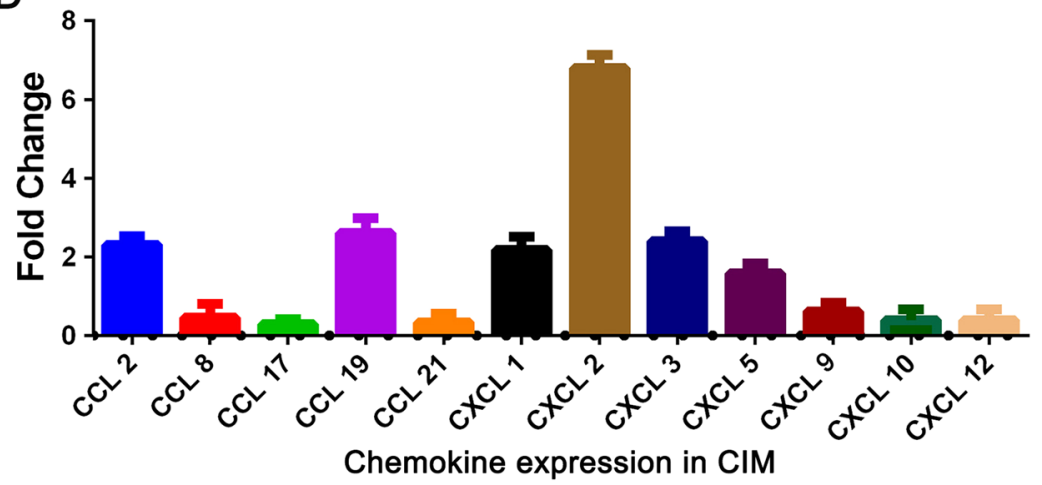

E

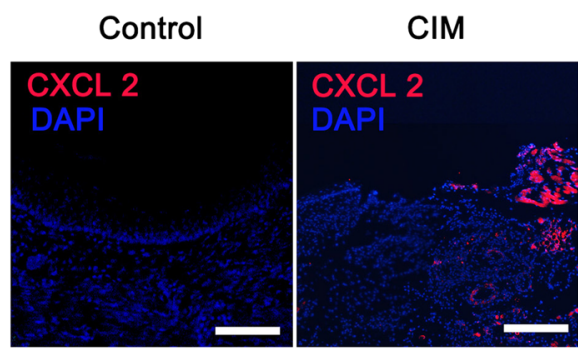

F

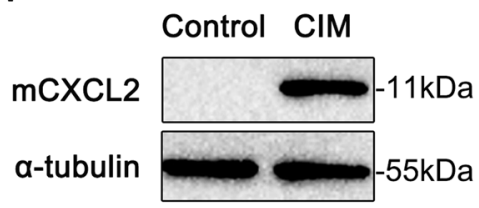

Fig. 1 (See legend on next page.)

potential of the transduced MSCs. A flow cytometry analysis indicated that $\mathrm{MSCs}{ }^{\mathrm{CXCR} 2}$ expressed the same pattern of stem cell surface markers as MSCs ${ }^{\mathrm{GFP}}$ (Supplementary Fig. 3C). Moreover, under mesenchymal lineage differentiation conditions, both $\mathrm{MSCs}{ }^{\mathrm{CXCR} 2}$ and MSCs $^{\text {GFP }}$ showed similar trilineage differentiation potential and exhibited osteogenic, adipogenic, and chondrogenic lineage phenotypes during the 2-to-4-week 
(see figure on previous page)

Fig. 1 CXCL2 is upregulated in radiation/chemical-induced mucositis. a The mRNA expression levels of various chemokines involved in radiation-induced tongue mucositis were analyzed by RT-qPCR. Samples were extracted from normal and inflamed tongues. The fold change represents the expression of each chemokine mRNA in inflamed tongues compared with normal tongues on day 7 after radiation. All the data are presented as the means \pm standard errors of the means (SEMs) $(n=6)$ for each group. $\mathbf{b}$ Immunofluorescence staining of CXCL2 (red) in normal and inflamed tongues on day 5 after radiation. The experiments were repeated three times in individual mice. Nuclei were visualized through DAPI staining (blue). Scale bar $=100 \mu \mathrm{m}$. c Total tissue lysates of normal and inflamed tongues were subjected to western blot analysis of mouse CXCL2 protein expression. The experiments were repeated three times, and a representative blot is shown. $\mathbf{d}$ The fold changes in the mRNA levels of various chemokines in chemical-induced mucositis were analyzed by RT-qPCR. Samples were extracted from normal and inflamed mucosal tissues on day 5 after chemical stimulation. The data are presented as the means \pm SEMs $(n=6)$ for each group. e Immunofluorescence staining for CXCL2 (red) in normal and inflamed mucosal tissues on day 5 after chemical stimulation. The experiments were repeated three times in individual mice. Nuclei were visualized through DAPI staining (blue). Scale bar $=100 \mu \mathrm{m}$. $\mathbf{f}$ Total tissue lysates from normal and inflamed mucosal tissues were subjected to western blot analysis of mouse CXCL2. RIM radiation-induced mucositis, CIM chemical-induced mucositis

differentiation period (Fig. 2e). A cytogenetic analysis

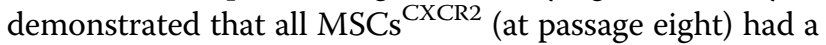
normal diploid chromosomal complement (Fig. 2f). Taken together, these results indicate that the transgenic modification of MSCs does not alter the intrinsic characteristics of MSCs.

\section{$\mathrm{MSCs}^{\mathrm{CXCR2}}$ exhibit enhanced migration potential in vitro and in vivo}

Chemokine axes mediate MSC migration to inflamed $\operatorname{sites}^{12,21}$. Hence, we used a chemotaxis assay to assess whether CXCR2-overexpressing MSCs exhibited enhanced targeting ability in vitro. The results showed that $\mathrm{MSCs}^{\mathrm{CXCR} 2}$, but not MSCs ${ }^{\mathrm{GFP}}$, strongly responded to $5 \mathrm{ng} / \mathrm{ml}$ human CXCL2 (hCXCL2) and $50 \mathrm{ng} / \mathrm{ml}$ murine CXCL2 (mCXCL2) (Fig. 3a). Additionally, the number of migrated MSCs ${ }^{\mathrm{CXCR} 2}$ increased nearly two-fold when the incubation time was extended from 6 to $12 \mathrm{~h}$ (Fig. 3b).

To determine whether MSCs ${ }^{\text {CXCR2 }}$ had an enhanced ability to target radiation-damaged tongues in vivo, we injected MSCs ${ }^{\text {CXCR2 } 2 \text { and MSCs }}{ }^{\text {GFP }}$ into RIM model mice through the tail vein on day 7 after radiation. Inflamed tongues were collected from each group on days 1 and 3 post-injection. An immunofluorescence staining analysis demonstrated that $\mathrm{MSCs}^{\mathrm{CXCR} 2}$ accumulated in the mucositis region in the RIM model. Interestingly, within 3 days of injection, the number of MSCs ${ }^{\mathrm{GFP}}$ declined sharply, and the number of MSCs ${ }^{\mathrm{CXCR} 2}$ decreased slightly (Fig. 3c, d). To further investigate whether MSCs ${ }^{\text {CXCR2 }}$ displayed enhanced targeting ability to CIM sites, we injected MSCs ${ }^{\mathrm{CXCR} 2}$ and MSCs ${ }^{\mathrm{GFP}}$ into CIM model mice. An immunostaining analysis indicated that the number of MSCs ${ }^{\text {CXCR2 }}$ was significantly higher than that of MSCs ${ }^{\text {GFP }}$ in the inflamed mucosa (Supplementary Fig. 4A, B). Altogether, these results indicate that CXCR2 overexpression enhances MSC targeting to RIM/CIM sites.

\section{$\mathrm{MSCs}^{\mathrm{CXCR2}}$ exhibit enhanced survival in RIM}

We inserted luciferase 2-monooxygenenase (luc) after the chemokine receptor in a lentiviral vector, placing both constructs under the control of the same promoter
(Supplementary Figs. 3A, B), and infected MSCs to allow their tracing after in vivo transplantation. Bioluminescence imaging (BLI) revealed a strong linear relationship $\left(r^{2}=0.992\right)$ between the number of MSCs and the average luminescence intensity (Fig. 4a, b), indicating that the number of MSCs can be determined by the luminescence intensity. Thus, to investigate MSC survival after systemic injection in mouse models, we examined the average luminescence intensity through BLI. The average luminescence intensity of both groups peaked 1 day after infusion. Although the luminescence intensity in the MSC $^{\text {GFP }}$ group decreased sharply within 3 days, the luminescence intensity in the MSC ${ }^{\mathrm{CXCR} 2}$ group remained steady for 3 days and was even observed after 7 days (Fig. 4c, d). These findings indicated that the overexpression of CXCR2 in MSCs not only enhanced their targeting ability to mucositis sites but also increased their survival rate.

To further verify that the overexpression of CXCR2 in MSCs increased their survival rate, we used an in vitro hydrogen peroxide $\left(\mathrm{H}_{2} \mathrm{O}_{2}\right)$ model as previously reported $^{22}$. Cell Counting Kit-8 (CCK-8) assays indicated that MSCs ${ }^{\text {CXCR2 }}$ exhibited increased survival rates 2 and $4 \mathrm{~h}$ after exposure to oxidative stress compared with MSCs $^{\text {GFP }}(P<0.05)$ (Fig. 4e). The PI3K/Akt and MAPK/ Erk signaling pathways have a large impact on cell survival and proliferation ${ }^{23}$. A western blot analysis indicated that the P-Akt and P-Erk $1 / 2$ levels were significantly increased in $\mathrm{MSCs}^{\mathrm{CXCR} 2}$ (Fig. 4f). These results indicated that CXCR2 enhances cell survival and proliferation, likely by activating the PI3K/Akt and MAPK/Erk signaling pathways.

\section{$\mathrm{MSCs}^{\mathrm{CXCR2}}$ notably attenuate RIM/CIM}

To investigate whether the enhanced migration of MSCs ${ }^{\text {CXCR2 }}$ would improve their beneficial effect on oral mucositis, we injected mice with $\mathrm{MSCs}^{\mathrm{GFP}}$ and $\mathrm{MSCs}^{\mathrm{CXCR} 2}$ on days 7 and 5 after radiation and chemical induction, respectively. Hematoxylin and eosin (HE) staining revealed that the group with radiation-induced ulcers exhibited a loss of filiform papillae, ulcerations, a 
A

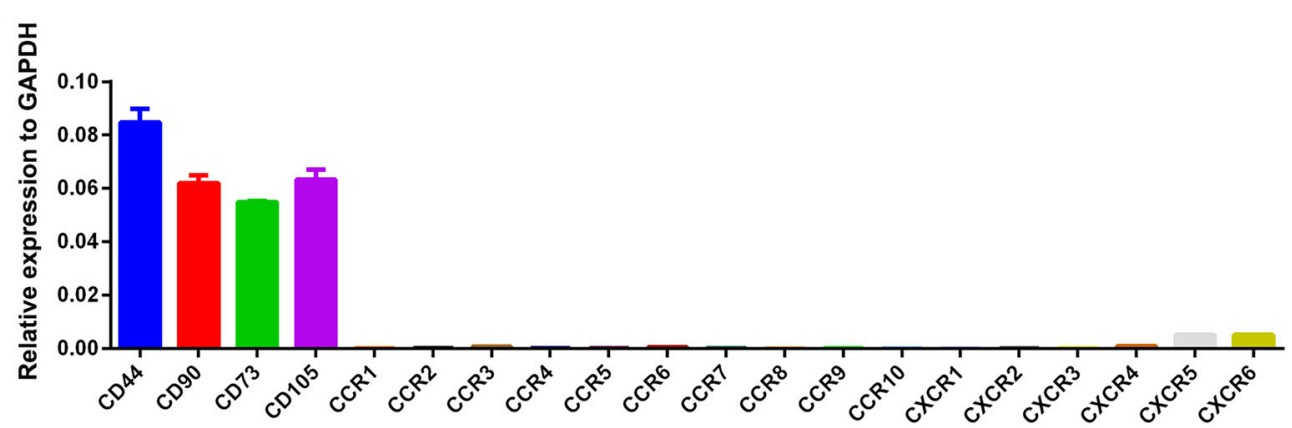

B

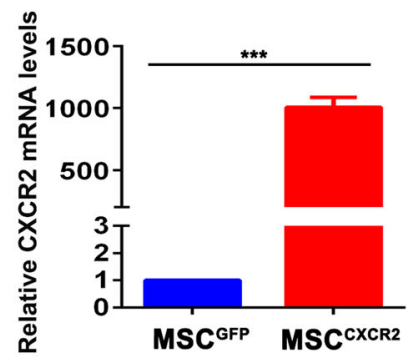

D

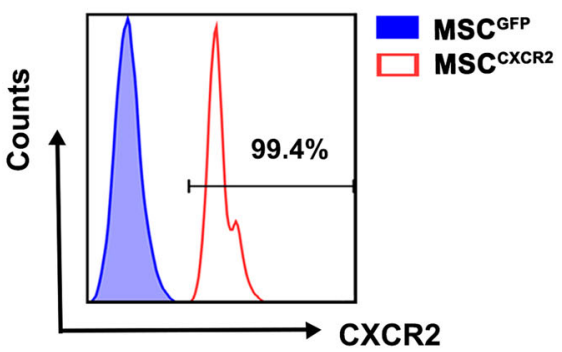

$\mathbf{F}$

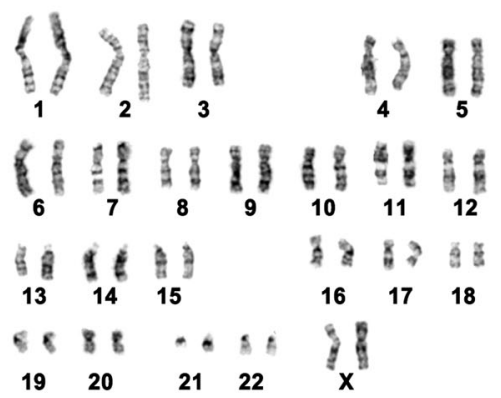

C

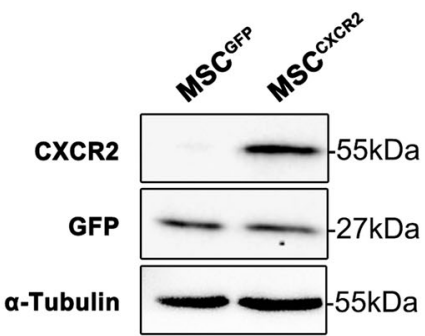

E

MSC GFP MSC CxCR2
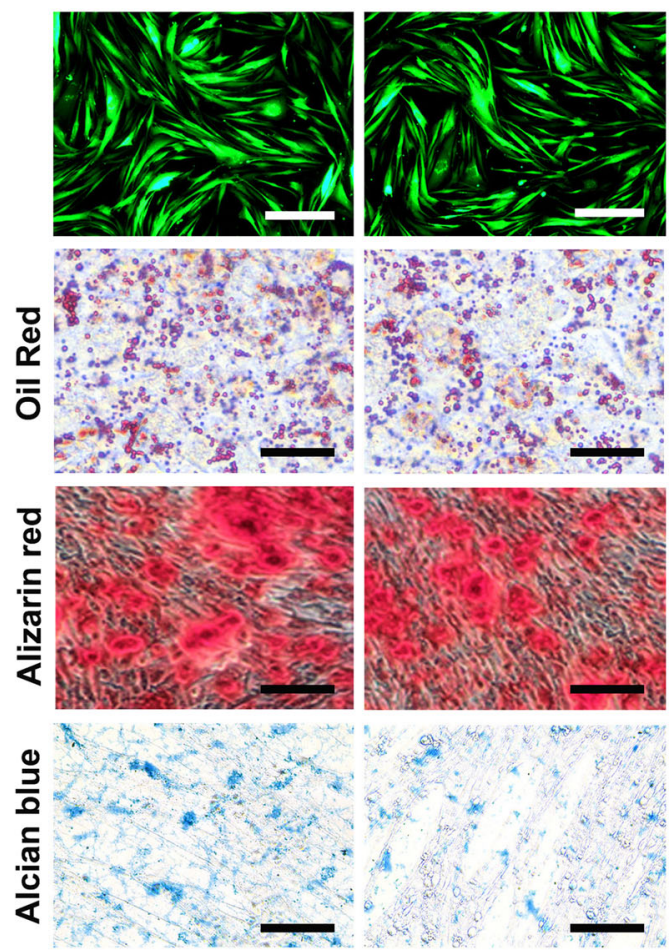

Fig. 2 CXCR2-overexpressing MSCs retain the characteristics of human MSCs. a Histograms representing the mRNA expression levels of C-C and $\mathrm{C}-\mathrm{X}-\mathrm{C}$ chemokine receptors from three independent samples of sixth-passage MSCs. MSC surface markers (CD44, CD90, CD73, and CD105) were detected as a positive control. The data are presented as the means \pm SEMs for each group ( $n=3, t$-test). $\mathbf{b}$ The expression levels of CXCR2 mRNA in $M S C s^{G F P}$ and MSCs ${ }^{C X C R 2}$ after gene transduction were analyzed via RT-qPCR. Glyceraldehyde 3-phosphate dehydrogenase (GAPDH) mRNA was detected as an internal control. The data are presented as the means \pm SEMs for each group ( $n=3, t$-test). c Total MSC GFP and MSC CXCR2 Iysates from three independent samples were subjected to western blot analysis of CXCL2 expression. $\mathbf{d}$ A flow cytometry analysis was used to detect CXCR2 protein on the surface of $\mathrm{MSCs}^{\mathrm{GFP}}$ and $\mathrm{MSCs}^{\mathrm{CXCR2}}$. Six independent samples were analyzed. e Representative images showing the trilineage differentiation potential of MSCs ${ }^{\text {CXCR2 }}$ and MSCs ${ }^{\text {GFP }}$ into adipocytes (oil red O), osteocytes (alizarin red), and chondrocytes (alcian blue). Scale bar $=20$ $\mu \mathrm{m}$. $\mathbf{f}$ Cytogenetic analysis of MSCs ${ }^{\mathrm{CXCR2}}$ at the eighth passage. The experiments were repeated three times 
A

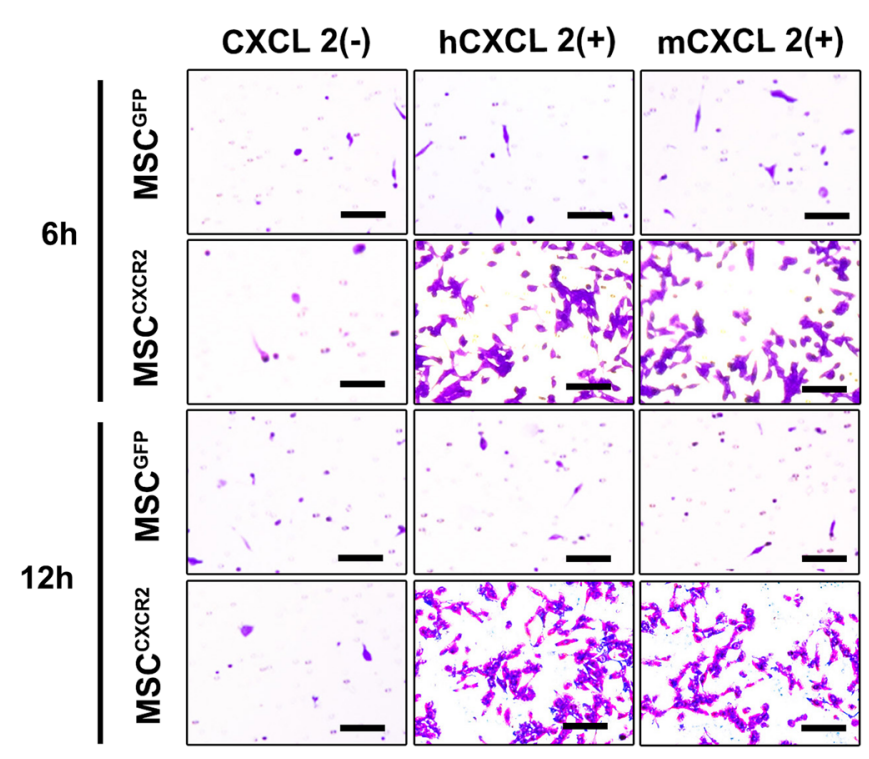

C
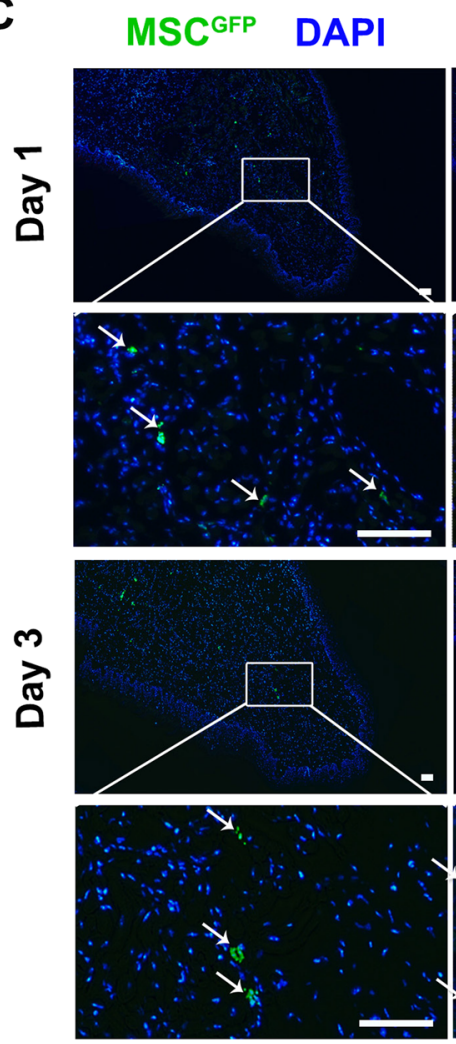

MSC CXCR2 DAPI
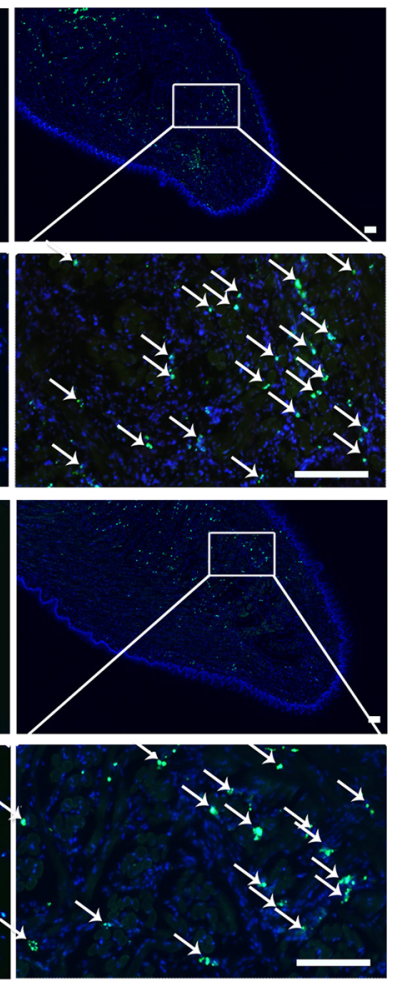

B

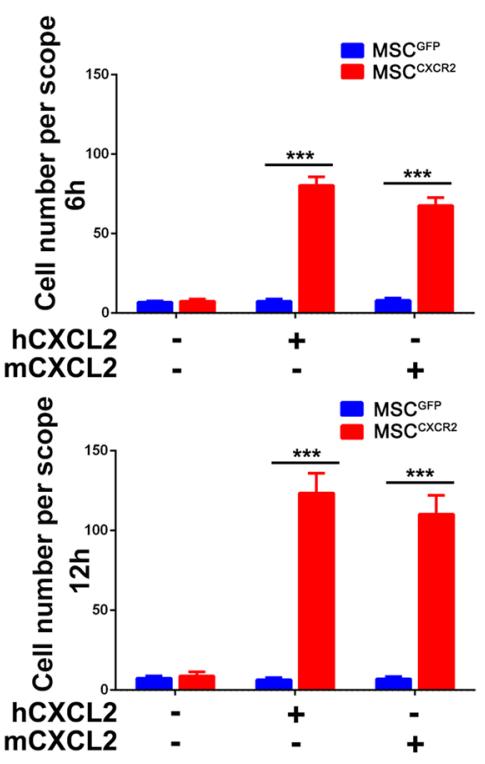

D

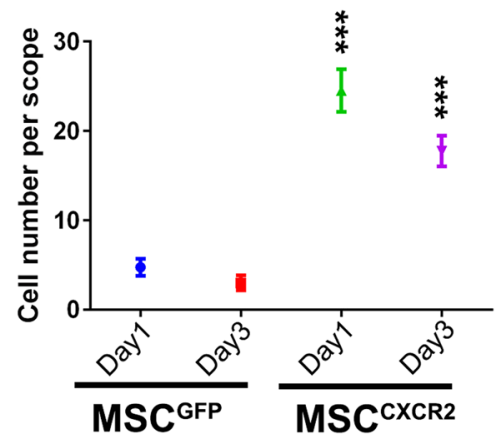

Fig. 3 MSCs ${ }^{\text {CXCR2 }}$ exhibit enhanced migration potential in vitro and in vivo. a In vitro migration of MSCS ${ }^{C X C R 2}$ and MSCS ${ }^{G F P}$ toward human $C X C L 2$ (hCXCL2) or murine CXCL2 (mCXCL2). Transwell filters were stained with $0.1 \%$ crystal violet and observed under a microscope. Scale bar $=25 \mu \mathrm{m}$. $\mathbf{b}$ The migrated MSCs after incubation for either 6 or $12 \mathrm{~h}$ were quantified in each microscopic field; ***P $<0.001$. c MSCs ${ }^{\text {CXR2 }}$ and MSCs ${ }^{\text {GFP }}$, both of which expressed GFP when injected into radiation-induced mucositis models, were examined by immunofluorescence staining on days 1 and 3 post-injection. Signals: GFP, green; DAPI, blue. Scale bar $=100 \mu \mathrm{m}$. d GFP-positive cells were quantified in each microscopic field of the mouse tongue. The data are presented as the means \pm SEMs for each group $\left(n=6, t\right.$-test). ${ }^{* * *} P<0.001$ for the RIM + MSC $^{\text {CXCR2 }}$ group compared with the RIM + MSC $^{\text {GFP }}$ group on the indicated day 
A

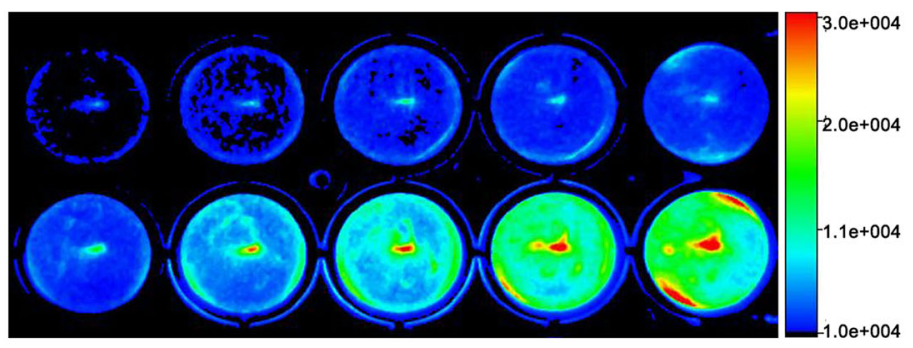

C

$\mathrm{MSC}^{\mathrm{GFP}}$

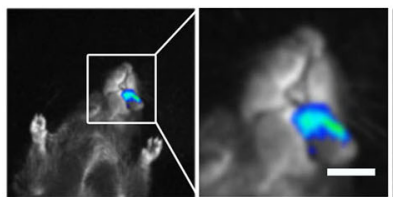

Day1

Day3

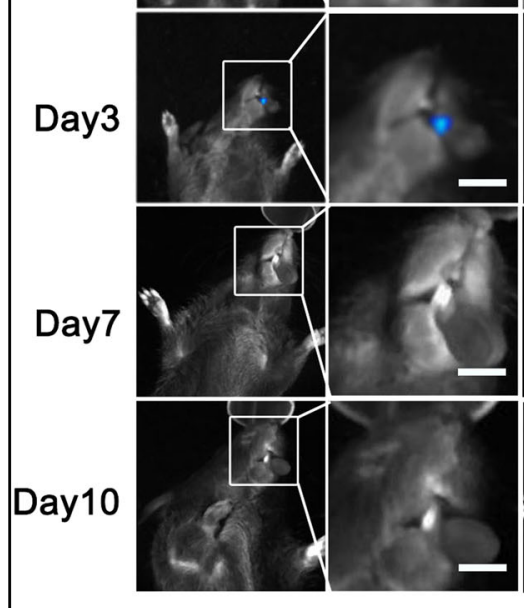

E

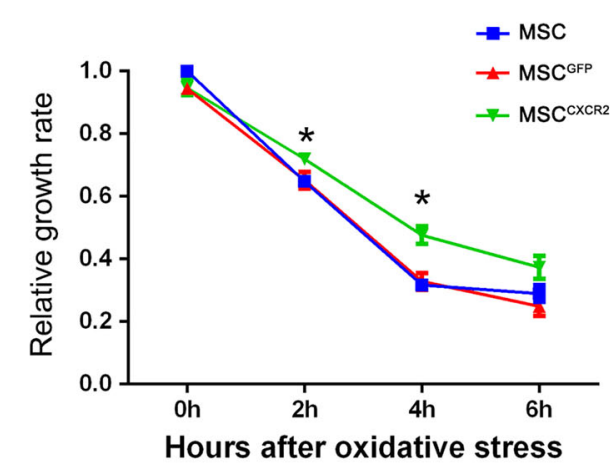

666.67

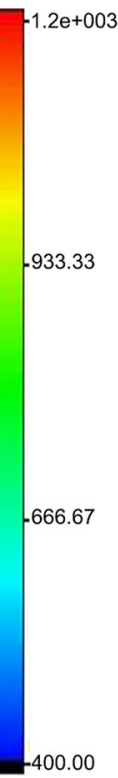

400.00

F
B

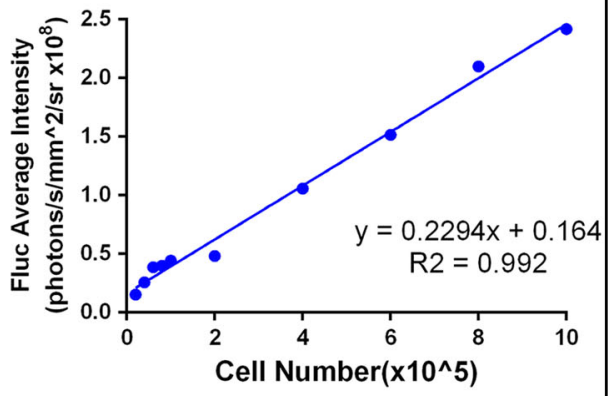

D

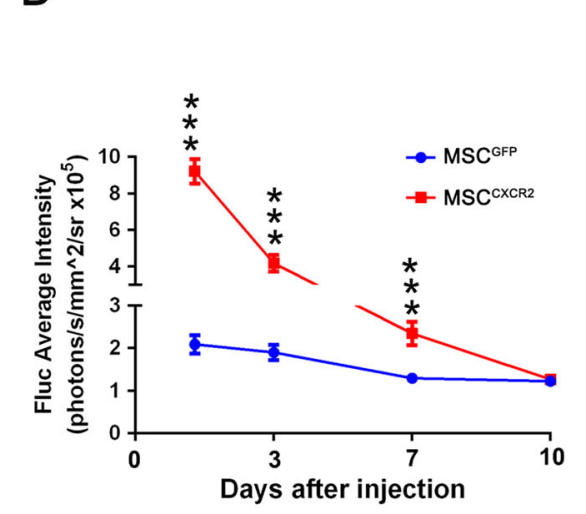

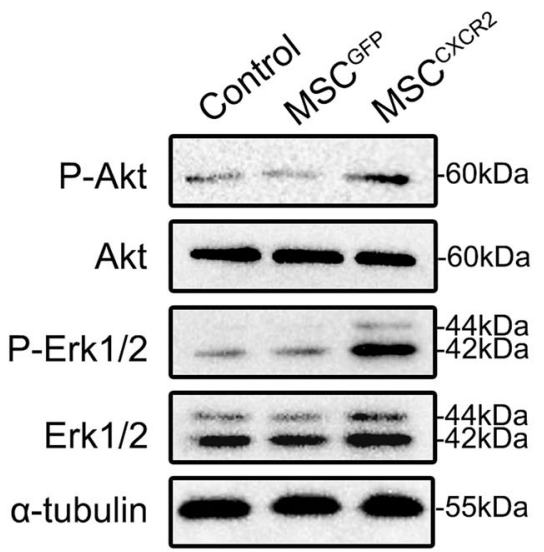

Fig. 4 MSCs ${ }^{C X C R 2}$ exhibit enhanced cell survival in radiation-induced mucositis. a In vitro bioluminescence imaging (BLI) of MSCS ${ }^{C X C R 2 ~ f r o m ~}$ groups with different cell numbers. The color scale bar represents the optical fluorescence intensity in photons/second/ $\mathrm{cm}^{2} / \mathrm{steradian}$ (photons/s/ $\left.\mathrm{cm}^{2} / \mathrm{sr}\right)$. $\mathbf{b}$ Linear correlation of the cell number with the luc-induced luminescence intensity $\left(r^{2}=0.992\right)$. $\mathbf{c}$ In vivo BLI representing MSC survival on days $1,3,7$, and 10 after injection. The luminescence intensity represents the number of living cells. Scale bar $=10 \mathrm{~mm}$. $\mathbf{d}$ Quantitative analysis of BLI on days $1,3,7$, and 10 after injection. The data are presented as the means \pm SEMs $(n=3)$. ${ }^{* *} P<0.001$. e CCK-8 assay showing the viability of MSCs $^{C X C R 2}$ and MSCs ${ }^{G F P} 2 \mathrm{~h}$ and $4 \mathrm{~h}$ after exposure to oxidative stress. The data are presented as the means \pm SEMs $(n=3)$ for each group and are representative of three independent experiments. ${ }^{*} P<0.05$. $\mathbf{f}$ Total MSC ${ }^{G F P}$ and MSC ${ }^{C X C R 2}$ lysates were subjected to western blot analysis of Akt and Erk1/2 phosphorylation. The experiments were repeated three times 
disrupted epithelium layer, inflammatory cell infiltration, and decreased mucosal thickness. The MSC ${ }^{\text {GFP }}$ group showed an increase in filiform papillae and decreased ulceration, but inflammatory cell infiltration was still observed in the submucosal area. However, extensive ulceration was absent in the $\mathrm{MSC}^{\mathrm{CXCR} 2}$ group, which exhibited the lowest level of inflammatory cell infiltration and increased thickness of the epithelium layer and mucosal lining (Fig. 5a). We then examined the maximum diameter of ulceration in RIM/CIM models to assess the severity of mucositis. Smaller ulceration diameters in the mouse mucosa were measured in the $\mathrm{MSC}^{\mathrm{CXCR} 2}$ group than in MSC ${ }^{\text {GFP }}$ group (Fig. 5b). Inflammatory cytokines, including tumor necrosis factor- $\alpha$ (TNF- $\alpha)$, interleukin- $1 \beta$ (IL-1 $\beta)$, IL-6, and IL-10, play important roles in wound repair ${ }^{4,16}$. Hence, we examined the levels of inflammatory cytokines after MSC treatment to assess the antiinflammatory effects of MSCs. A RT-qPCR analysis demonstrated that the mRNA expression of proinflammatory cytokines in the mouse mucosa was significantly downregulated in the MSC ${ }^{\mathrm{CXCR} 2}$ group but only slightly reduced in the $\mathrm{MSC}^{\mathrm{GFP}}$ group (Fig. 5c). These results indicate that MSCs ${ }^{\text {CXCR2 }}$ can markedly attenuate oral mucositis, in part due to their anti-inflammatory effects.

\section{MSCs accelerate mucositis recovery by decreasing radiation-induced ROS production}

RIM is initially caused by radiogenic $\operatorname{ROS}^{4,24}$. Because MSCs have great anti-oxidation potential ${ }^{25,26}$, we wondered whether MSCs promoted ulcer healing by protecting tissue cells from ROS-induced damage. The staining of inflamed tongue tissues with the superoxidesensitive fluorescent dye dihydroethidium (DHE) revealed that both $\mathrm{MSC}^{\mathrm{GFP}}$ and $\mathrm{MSC}^{\mathrm{CXCR} 2}$ treatment exerted benefits by reducing the cellular ROS levels. However, MSC $^{\text {CXCR2 }}$ treatment had the greatest effect on decreasing the cellular ROS levels among all groups in vivo (Fig. 6a). We further applied CellROX ${ }^{\mathrm{TM}}$ for detection of the radiogenic ROS levels in primary tongue epidermal and fibroblast cells after treatment with 4Gy of radiation in vitro. Based on immunofluorescence staining, the radiogenic ROS levels in primary tongue cells were attenuated by co-culture with MSCs ${ }^{\text {CXCR2 }}$ or MSCs ${ }^{\text {GFP }}(P<$ $0.05)$ (Fig. 6b). Furthermore, a flow cytometry analysis of the intracellular redox status confirmed that primary tongue epidermal or fibroblast cells co-cultured with MSCs exhibited less intracellular ROS generation than mono-cultured cells (Fig. 6c). In addition, an oxidative environment contributes to cell death, which is also responsible for ulcer healing delays ${ }^{22,27,28}$. Therefore, we used an $\mathrm{H}_{2} \mathrm{O}_{2}$-induced cell death model to determine whether MSCs protect tongue epidermal or fibroblast cells from cell death in an oxidative environment. Cell apoptosis was measured via Annexin V/propidium iodide (PI) staining and flow cytometry. The apoptosis of primary tongue cells subjected to co-culture with MSCs was significantly decreased compared with mono-cultured MSCs (Supplementary Fig. 5A, B). Taken together, these findings indicate that MSCs accelerate mucositis recovery, likely by decreasing radiogenic ROS production and protecting tongue cells from cell death.

\section{Discussion}

Oral RIM is one of the most common complications of radiotherapy among patients with head and neck cancer. However, traditional treatments for severe mucositis are limited and cannot prevent ulcer recurrence. Here, we report an innovate and highly efficient treatment, namely, the systematic transplantation of MSCs with an enhanced targeting capacity to mucositis sites. This study sheds new light on the treatment of RIM and provides groundwork for the clinical application of MSC-based therapy for mucositis.

Due to their anti-inflammatory and immunomodulatory capabilities, MSCs have therapeutic potential for the treatment of inflammatory diseases ${ }^{6,9}$. However, systemically infused MSCs rarely home to inflamed mucosa in the orofacial region. MSC targeting depends on the interactions between chemokine receptors and specific chemokines from inflamed sites $^{12,21}$. A RT-qPCR analysis revealed that CXCL2 was the most highly expressed chemokine in the inflamed mucosa in oral RIM mouse models. Recent studies have reported that the overexpression of corresponding chemokine receptors in MSCs can enhance their targeting ability to inflamed sites. For example, CCR1, CXCR4, and CCR7 overexpression has been shown to enhance MSC targeting to injured myocardium $^{29}$, ischemic myocardium ${ }^{30}$, and secondary lymphoid organs ${ }^{31}$, respectively. Notably, our chemotaxis assays demonstrated that MSCs overexpressing CXCR2 had enhanced migratory ability when exposed to CXCL2 in vitro. Furthermore, immunostaining and BLI analyses revealed that MSCs ${ }^{\text {CXCR2 }}$ exhibited enhanced targeting to the inflamed mucosa. Interestingly, we also observed that CXCR2 overexpression prolonged MSC survival in the inflamed mucosa, and this effect is likely mediated by PAkt and P-Erk1/2 upregulation.

Subsequently, we exploited the therapeutic potential of MSC $^{\text {CXR2 transplantation. A HE staining analysis }}$ demonstrated that mice that received MSCs ${ }^{\text {CXCR2 }}$ displayed enhanced epithelial integrity in the mucosa compared with those that received MSCs ${ }^{\mathrm{GFP}}$. Furthermore, the ulceration diameters in the MSC ${ }^{\mathrm{CXCR} 2}$-treated group were significantly smaller than those in the MSC $^{\text {GFP }}$ treated control group. Together, these results suggest that

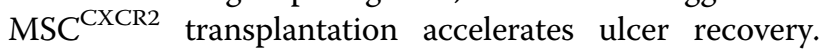
Conventional treatments, such as pain management and 


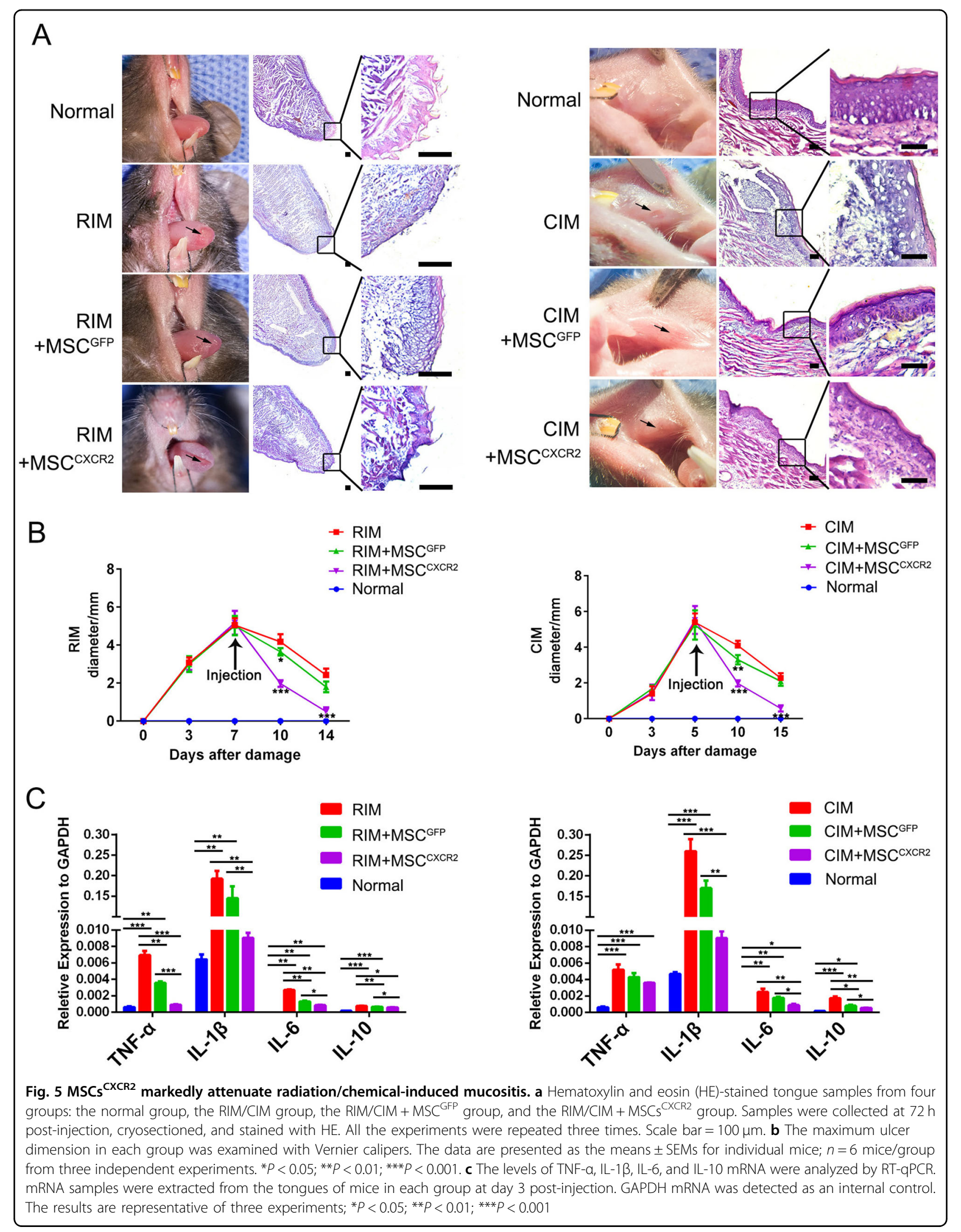



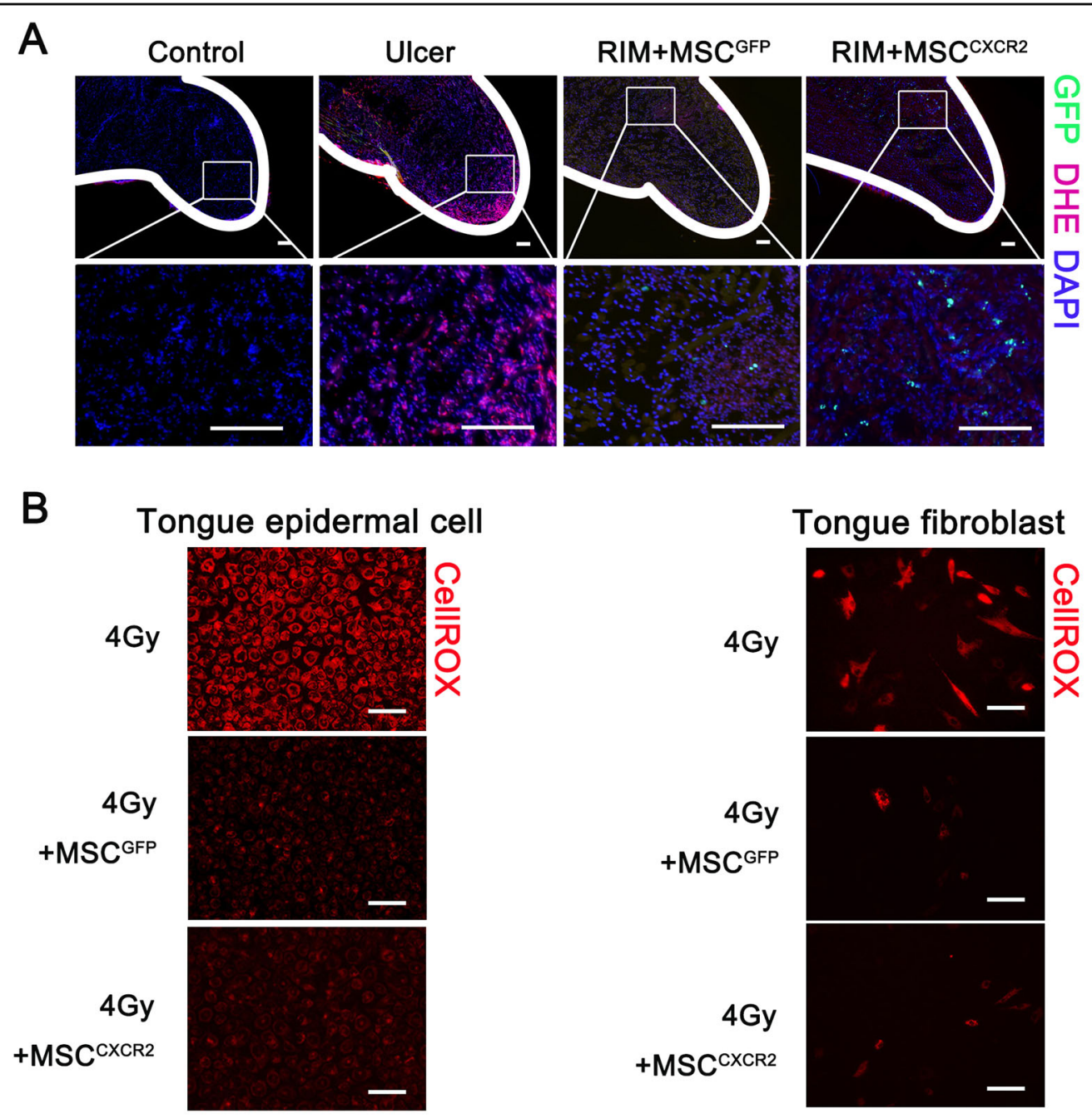

C Tongue epidermal cell
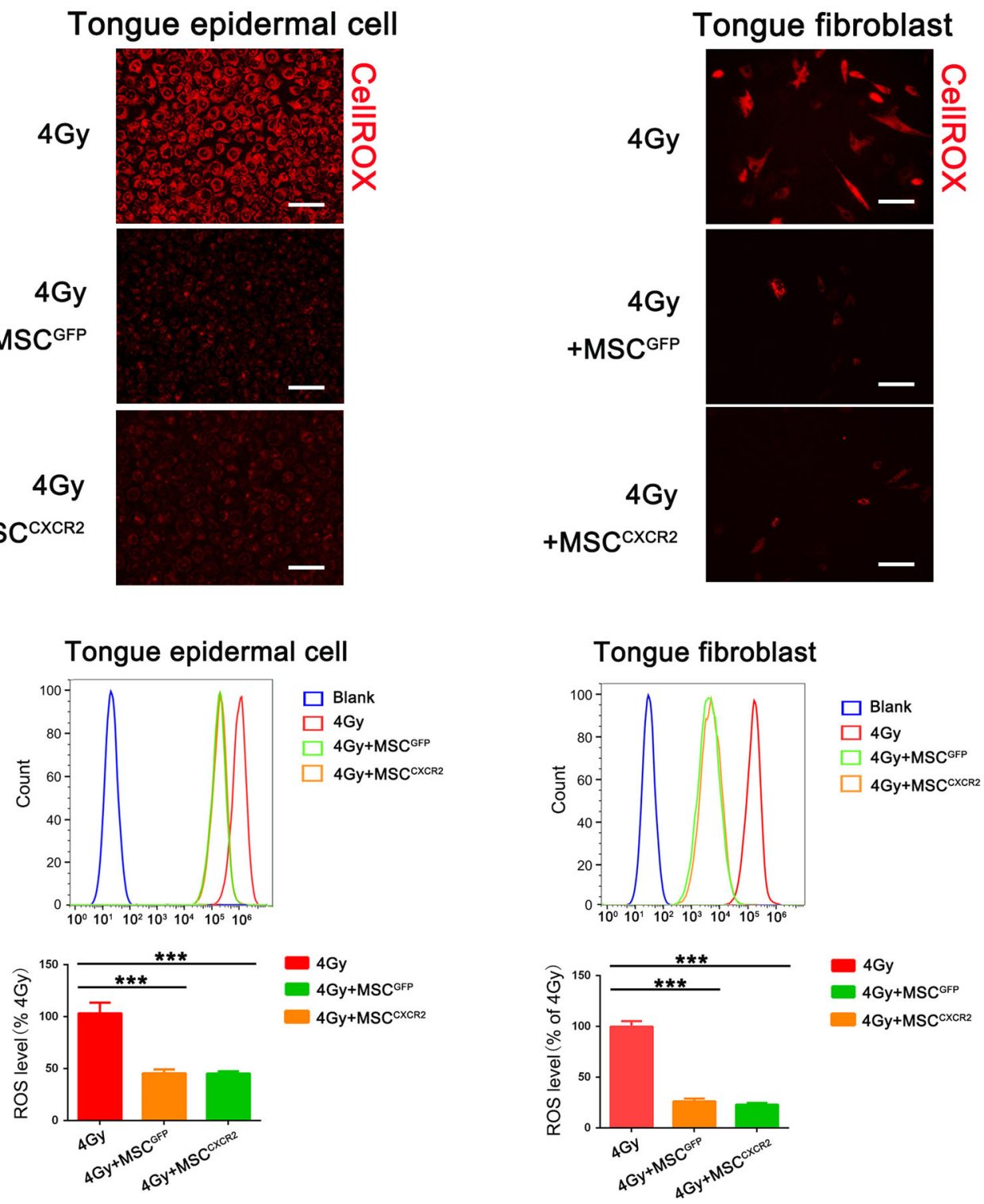

Fig. 6 (See legend on next page.) 
(see figure on previous page)

Fig. 6 MSCs accelerate mucositis recovery by decreasing radiation-induced ROS production. a Representative images of DHE staining. Samples were collected on day 3 post-injection, $n=6$ mice/group from three independent experiments. The tissue ROS levels were detected through DHE staining (red). Signals: GFP, green; DAPI, blue. Scale bar $=100 \mu \mathrm{m}$. b Primary tongue epithelial cells and tongue fibroblasts were exposed to $4 \mathrm{~Gy}$ of radiation and then cultured with or without MSCs for $48 \mathrm{~h}$. The experiments were repeated three times. The cellular ROS levels were visualized with CellROX staining (red). Scale bar $=100 \mu \mathrm{m}$. c Density plot of the total ROS levels in primary tongue epithelial cells and tongue fibroblasts that were mono-cultured in suspension or co-cultured with MSCs in transwell chambers. The data are presented as the means \pm SEMs ( $n$ =3) for each group $\left({ }^{* *} P<0.001\right.$; $t$-test $)$

nutrition support therapy, have limited healing benefits in mucositis $^{1,32}$. Additionally, due to conflicting evidence, no specific guideline regarding anti-inflammatory agents for RIM can be found among the Multinational Association of Supportive Care in Cancer (MASCC) clinical practice guidelines ${ }^{1,5}$. Recently, growth factors have been applied for the prevention of oral RIM, such as FDA-approved palfermin (recombinant human KGF) ${ }^{1,33}$. These factors play a marked role in the proliferation of various cell types, including endothelial cells, fibroblasts, vascular smooth muscle cells, and keratinocytes, thus promoting mucosa recovery ${ }^{34}$. However, because growth factors also promote angiogenesis in tumors ${ }^{35,36}$, the administration of growth factors might increase the risk of head and neck cancer recurrence, which hinders their application. In addition, prolonged pro-inflammatory cytokine production greatly impacts the development, metastasis, and recurrence of cancer ${ }^{37}$. However, growth factors can hardly alleviate mucosal inflammation. Interestingly, we found that implanted MSCs downregulated proinflammatory cytokines, including TNF- $\alpha$, IL- $1 \beta$, and IL6. We further found that MSCs significantly decreased the cellular ROS levels both in vivo and in vitro. ROS generation is widely acknowledged to be the primary event in most mucositis-causing pathways ${ }^{4,24}$. For example, ROS lead to DNA damage, cell death, and upregulation of various inflammation-related signaling pathways ${ }^{4,22}$. Hence, the effects of MSCs on alleviating inflammation in mucositis are partially due to their anti-oxidant capability.

The mechanism of how transplanted MSCs promote tissue repair have long been debated ${ }^{38-40}$. MSCs accelerate tissue repair probably in three ways: by direct differentia$\operatorname{tion}^{38}$, direct cellular interactions ${ }^{39}$, or secretion of soluble factors $^{40}$. Our BLI analysis showed that MSCs were not detected in the newly formed epithelium layer and mucosal lining 10 days after injection in the RIM mouse models, suggesting that MSCs may not accelerate ulcer healing via directly generating tongue epithelial cells or fibroblast cells. In addition, we found that MSCs significantly decreased the ROS levels of tongue epithelial cell or those of the fibroblast cell in both transwell and direct co-culture systems with no significant difference between the two co-culture systems (data not show). Because MSCs can secrete factors in both co-culture systems while exhibited similar anti-oxidant effects in the two systems,
MSCs might decrease the cellular ROS levels not via direct cellular interactions. Growing evidences indicate that MSCs can produce soluble factors, especially antioxidant factors, to promote tissue repair via their secretome ${ }^{40,41}$. Our study showed that tongue epithelial cells or fibroblast cells exhibited reduced cellular ROS levels when cocultured with MSCs via a transwell system. We deduced that the anti-oxidative capability of MSCs mainly lies in their secretion of factors. However, these anti-oxidative secretion factors of MSCs needs to be further identified.

In summary, the transplantation of CXCL2overexpressing MSCs promotes ulcer healing. Because radiotherapy is one of the most widely used antineoplastic treatments for head and neck cancer, reducing the harmful side effects of radiation will greatly impact patient quality of life. Thus, this innovative strategy to reduce the side effects of radiotherapy and improve patient tolerance to treatment is promising for clinical application. In addition, MSCs are attractive candidates for the delivery of anti-tumor reagents or cytokines in human malignancies. MSC treatment will lead to a safer and more effective clinical therapy for tumors and reduce radiotherapy or chemotherapy complications. However, further investigations are needed to establish the longterm safety and effects of MSC transplantation therapy.

\section{Materials and methods}

\section{Animal experiments}

Male mice (C57) were purchased from the Animal Center of Sun Yat-sen University (Guangzhou, China). All the experiments were approved by the Animal Care and Use Committee of Sun Yat-sen University (IACUC-DB16-1215). Animal models were established as previously described $^{42}$. The tongue was extended out of the mouth and exposed to $16 \mathrm{~Gy}$ (a dose of $1.6 \mathrm{~Gy} / \mathrm{min}$ ) of radiation through a $10-\mathrm{mm}$-diameter hole to establish RIM. Oral ulcers were chemically induced by placing a $3 \times 3-\mathrm{mm}$ round filter paper soaked with $70 \%$ acetic acid on the buccal mucosa for $30 \mathrm{~s}$. The results are representative of three independent experiments (six animals per group). Detailed information is shown in Supplementary Fig. 1.

\section{Isolation and characterization of MSCs}

MSCs were obtained from healthy donors who provided informed consent. MSCs were isolated from the bone 
marrow using our previously reported method ${ }^{13}$. Briefly, bone marrow cells were separated by Ficoll-Paque (1.077 $\mathrm{g} / \mathrm{ml}$; Amersham Biosciences, Uppsala, Sweden) density gradient centrifugation. MSCs were maintained in growth medium consisting of low-glucose Dulbecco's modified Eagle's medium (L-DMEM; HyClone, Logan, UT, USA) and $10 \%$ fetal bovine serum (FBS; Gibco, Grand Island, NY, USA). The MSCs were then seeded into T25 cell culture flasks at a density of $1 \times 10^{5}$ cells $/ \mathrm{ml}$. The cultureexpanded MSCs exhibited surface expression of CD44, CD73, CD90, and CD105 (MSC markers) but not CD34 or CD45 (hematopoietic markers). After the fourth passage, the capacity of MSCs to differentiate into multiple lineages was confirmed by their differentiation to osteoblasts, chondrocytes, or adipocytes, which was performed using previously described methods ${ }^{13}$.

\section{RNA isolation, reverse transcription, and RT-qPCR}

Total RNA was extracted using TRIzol (Invitrogen, Carlsbad, CA, USA) according to the manufacturer's instructions. Reverse transcription was performed using ReverTra Ace (Toyobo Co, Ltd, Osaka, Japan), and RTqPCR was performed using SYBR PCR Master Mix (Roche, Indianapolis, IN, USA). Three independent experiments were performed. The signals were detected using a Light Cycler 480 Detection System (Roche, Sweden). The primer sequences are listed in Supplementary Table 1.

\section{Western blot analysis}

Cells and tissues subjected to the indicated treatments were prepared, lysed in radioimmunoprecipitation assay (RIPA) buffer, and centrifuged at $10,000 \mathrm{rpm}$ and $4{ }^{\circ} \mathrm{C}$ for $10 \mathrm{~min}$. The proteins were separated by sodium dodecyl sulfate-polyacrylamide gel electrophoresis (SDS-PAGE) and transferred to a poly vinylidene fluoride (PVDF) membrane (Millipore, Bedford, MA, USA). The membrane was then blocked with $5 \%$ milk for $1 \mathrm{~h}$, incubated with the appropriate primary antibodies at $4{ }^{\circ} \mathrm{C}$ overnight, and then incubated with horseradish peroxidaseconjugated secondary antibodies for $1 \mathrm{~h}$ at room temperature. Bands were detected with a chemiluminescence kit (Millipore). The primary and secondary antibodies are listed in Supplementary Table 2.

\section{Immunofluorescence staining}

The samples were embedded in the Tissue-Tek optimum cutting temperature (OCT) compound (Miles Scientific, Naperville, IL, USA) and frozen in a frozen slicer (Leica CM1900, Germany). The sections (5 mm thick) were placed on glass slides (Bio-Optica SpA, Milano, Italy), blocked with PBS-Tween $0.05 \%$ plus $0.5 \%$ FBS for $30 \mathrm{~min}$, and successively incubated with the appropriate primary antibodies overnight at $4{ }^{\circ} \mathrm{C}$ and secondary antibodies for $30 \mathrm{~min}$ at room temperature.

\section{Lentiviral vector construction and infection}

Lentiviral vectors expressing the CXCR2 gene were constructed by and purchased from Hanheng Biotechnology (Shanghai, China) (Supplementary Fig. 3A). For lentiviral infection, the optimal multiplicity of infection (MOI) was 50 (50 viral particles for each cell), which was determined in our previous experiments. According to the manufacturer's instructions, the cells were incubated with the virus overnight, and this solution was then removed and replaced with new complete medium.

\section{Flow cytometry}

The expression levels of CXCR2 and GFP on MSCs were analyzed by flow cytometry (FACScan; Becton Dickinson, San Diego, CA, USA) as previously described $^{43}$. Briefly, the MSCs were incubated for $30 \mathrm{~min}$ with the appropriate antibody in the dark at $4{ }^{\circ} \mathrm{C}$ and then analyzed by flow cytometry. CXCR- and GFP-positive cells were used for further study. Each analysis was performed with at least three independent experiments. The data were analyzed with FlowJo7.5 (Treestar, Ashland, OR, USA) or Kaluza (Beckman Coulter, Krefeld, Germany) software.

\section{BLI}

BLI of luc is a reliable noninvasive imaging tool used to quantitatively monitor MSC survival and distribution with high sensitivity. Briefly, after anesthetizing the mice via an intraperitoneal injection of $2 \%$ pentobarbital $(5 \mu \mathrm{l} /$ g) or inhalation of isoflurane anesthesia, the reporter probe D-luciferin (Invitrogen) was injected into the mouse model $(1500 \mathrm{mg} / \mathrm{kg})$, and the tongue was removed from the mouth, placed as close as possible to the detector and imaged for $1 \mathrm{~min}$ using a Bruker Xtreme imaging system (Bruker, Karlsruhe, Germany). Signals (photons $/$ second $/ \mathrm{cm}^{2} /$ steradian) from a fixed region of interest (ROI) were evaluated according to the manufacturer's instructions.

\section{CCK-8 assay}

The CCK- 8 assay was performed according to the manufacturer's instructions (Dojin Laboratories, Kumamoto, Japan). Briefly, $100 \mu \mathrm{l}$ of cell suspension was dispensed (5000 cells/well) into a 96-well plate. The plate was pre-incubated for $24 \mathrm{~h}$ in growth medium $\left(37^{\circ} \mathrm{C}, 5 \%\right.$ $\mathrm{CO}_{2}$ ), and $10 \mu \mathrm{l}$ of various concentrations of toxicant $\left(\mathrm{H}_{2} \mathrm{O}_{2}\right)$ were added to the culture medium in the plate. The plate was then incubated for an appropriate length of time in the incubator. CCK-8 solution $(10 \mu \mathrm{l})$ was added to each well of the plate, and the plate was incubated for $1-4 \mathrm{~h}$ in the incubator. The absorbance at $450 \mathrm{~nm}$ was measured using a microplate reader (Tecan, Infinite $\mathrm{f} 200$ PRO, Switzerland). The relative growth rate (RGR) was 
calculated using the following formula:

$R G R=\frac{O D \text { value (experimental group })-O D \text { value }(\text { blank control })}{O D \text { value (negative control })-O D \text { value }(\text { blank control })}$.

\section{Migration assays}

Migration was assessed using a transwell chamber system with 8 - $\mu \mathrm{m}$-pore filters (PIEP12R48, Millipore). Each upper chamber was loaded with serum-starved MSCs $\left(2 \times 10^{5}\right.$ per well), and each lower chamber was loaded with $500 \mu \mathrm{l}$ of serum-free medium with or without hCXCL2 (5 ng/ml, Peprotech) or mCXCL2 (50 $\mathrm{ng} / \mathrm{ml}$, Peprotech). After a 6- to 12 -h incubation at $37^{\circ} \mathrm{C}$ in $5 \% \mathrm{CO}_{2}$, the cells remaining on the upper surfaces were removed with a cotton swab, and the filters were fixed and stained with $0.1 \%$ crystal violet. Cells that migrated to the lower surface were counted under a microscope

\section{In vivo distribution of transplanted MSCs}

$\mathrm{MSCs}^{\mathrm{CXCR} 2}$ and MSCs ${ }^{\mathrm{GFP}}$ were examined on days 1 and 3 post-injection in oral RIM/CIM models. Cryosections were prepared and counterstained with $1.0 \mu \mathrm{g} / \mathrm{ml}$ DAPI in PBS for $3 \mathrm{~min}$ at room temperature in the dark.

\section{Primary tongue cell culture}

Tongue epithelial cells and fibroblasts were prepared and maintained as previously described ${ }^{44,45}$. Briefly, both cell types were isolated from mouse tongue tissue specimens. Tongue epithelial tissues and connective tissues were dissociated with $4 \%$ dispase and $4 \%$ collagenase I (37 ${ }^{\circ} \mathrm{C}$ for $1 \mathrm{~h}$ or $4{ }^{\circ} \mathrm{C}$ overnight). The tongue epithelial tissues could then easily be separated from whole tissues with forceps. Tongue epithelial cells were derived from epithelial tissues, and fibroblasts were derived from connective tissues. High-glucose DMEM and DMEM/F12 with 10\% FBS were used as the growth media for the primary cultures of epithelial cells and fibroblasts, respectively. The primary cells were plated at a density of $1 \times 10^{5}$ cells per 25 -mm culture dish. Third-passage cells were used in the experiments.

\section{ROS assessment}

The levels of total intracellular ROS and mitochondrial ROS were detected using the fluorescent probe CellROX Deep Red (Life Technologies, Carlsbad, CA, USA), and the fluorescence intensity was measured by flow cytometry. In addition, DHE was used to evaluate the in situ formation of $\mathrm{O}_{2}{ }^{-}$according to the manufacturer's instructions. Briefly, tongue sections were incubated with DHE (DHE, 1:1000 dilution, National Diagnostics, Atlanta, GA, USA) for $5 \mathrm{~min}$ at $37^{\circ} \mathrm{C}$. The tissue sections were visualized under a fluorescence microscope with a 590-nm wavelength filter.

\section{Flow cytometry analysis of apoptosis}

Primary tongue epithelial cells and fibroblasts were treated with $\mathrm{H}_{2} \mathrm{O}_{2}$ for $6 \mathrm{~h}$ and then cultured with or without MSCs for $48 \mathrm{~h}$. The cells were digested, monitored, and then stained for $15 \mathrm{~min}$ with Annexin V and PI (Biosci Biotech, Shanghai, China) in binding buffer according to the manufacturer's instructions. The apoptotic population was assessed by flow cytometry. Annexin $\mathrm{V}+/ \mathrm{PI}-$ cells represent early apoptotic cells, whereas Annexin $\mathrm{V}+/ \mathrm{PI}+$ cells are late apoptotic cells.

\section{Statistics}

All the data are presented as the means \pm SEMs from at least three independent experiments. Comparisons between groups were performed using Student's $t$-test or one-way analysis of variance (ANOVA) with a Newman-Keuls post hoc comparison. $P<0.05$ was considered statistically significant. All the statistical analyses were performed with SPSS version 19.0 (SPSS Inc., Chicago, IL, USA).

\section{Acknowledgements}

This work was supported by grants from the International Cooperation Project of Science and Technology in Guangdong Province (2016B050502008); the National Natural Science Foundation of China $(81670984,81700959,51702371$, 81702325); the Science and Technology Program of Guangzhou (201604020008); the Guangdong Natural Science Foundation (2017A030310565, 2015A030310079); and the China Postdoctoral Science Foundation (2016M602583, 2017T100657).

\section{Author details}

${ }^{1}$ Guangdong Provincial Key Laboratory of Stomatology, Department of Operative Dentistry and Endodontics, Guanghua School of Stomatology, Sun Yat-sen University, Guangzhou, China. ${ }^{2}$ The Key Laboratory for Stem Cells and Tissue Engineering, Center for Stem Cell Biology and Tissue Engineering, Ministry of Education, Sun Yat-sen University, Guangzhou, China. ${ }^{3}$ Department of Gastrointestinal Surgery of the First Affiliated Hospital of Sun Yat-sen University, Guangzhou, China. ${ }^{4}$ Department of Andrology, The First Affiliated Hospital, Sun Yat-sen University, Guangzhou, China. ${ }^{5}$ Department of

Prosthodontics, Hospital of Stomatology, Institute of Stomatological Research, Guanghua School of Stomatology, Sun Yat-sen University, Guangzhou, China

\section{Conflict of interest}

The authors declare that they have no conflict of interest.

\section{Publisher's note}

Springer Nature remains neutral with regard to jurisdictional claims in published maps and institutional affiliations.

Supplementary Information accompanies this paper at (https://doi.org/ 10.1038/s41419-018-0310-x).

Received: 31 October 2017 Revised: 26 December 2017 Accepted: 8 January 2018

Published online: 14 February 2018

\section{References}

1. Lalla, R. V. et al. MASCC/ISOO clinical practice guidelines for the management of mucositis secondary to cancer therapy. Cancer 120, 1453-1461 (2014).

2. Rodriguez-Caballero, A. et al. Cancer treatment-induced oral mucositis: a critical review. Int. J. Oral. Maxillofac. Surg. 41, 225-238 (2012). 
3. Sciubba, J. J. \& Goldenberg, D. Oral complications of radiotherapy. Lancet Oncol. 7, 175-183 (2006).

4. Sonis, S. T. et al. Perspectives on cancer therapy-induced mucosal injury: pathogenesis, measurement, epidemiology, and consequences for patients. Cancer 100, 1995-2025 (2004).

5. Nicolatou-Galitis, O. et al. Systematic review of anti-inflammatory agents for the management of oral mucositis in cancer patients. Support Care Cancer 21, 3179-3189 (2013).

6. Griffin, M. D. et al. Concise review: adult mesenchymal stromal cell therapy for inflammatory diseases: how well are we joining the dots? Stem Cells $\mathbf{3 1}$, 2033-2041 (2013)

7. Phinney, D. G. \& Prockop, D. J. Concise review: mesenchymal stem/multipotent stromal cells: the state of transdifferentiation and modes of tissue repair--current views. Stem Cells 25, 2896-2902 (2007).

8. Moon, H. H. et al. MSC-based VEGF gene therapy in rat myocardial infarction model using facial amphipathic bile acid-conjugated polyethyleneimine. Biomaterials 35, 1744-1754 (2014).

9. Chang, P. Y., Qu, Y. Q., Wang, J. \& Dong, L. H. The potential of mesenchymal stem cells in the management of radiation enteropathy. Cell Death Dis. $\mathbf{6}$, e1840 (2015).

10. Khaldoyanidi, S. Directing stem cell homing. Cell Stem Cell 2, 198-200 (2008).

11. Karp, J. M. \& Leng, T. G. Mesenchymal stem cell homing: the devil is in the details. Cell Stem Cell 4, 206-216 (2009).

12. Griffith, J. W., Sokol, C. L. \& Luster, A. D. Chemokines and chemokine receptors: positioning cells for host defense and immunity. Annu Rev Immunol. 32, 659-702 (2014).

13. Zhang, $X$. et al. CXCR5-overexpressing mesenchymal stromal cells exhibit enhanced homing and can decrease contact hypersensitivity. Mol. Ther. 25, 1434-1447 (2017).

14. Ruster, B. et al. Mesenchymal stem cells display coordinated rolling and adhesion behavior on endothelial cells. Blood 108, 3938-3944 (2006).

15. Rombouts, W. J. \& Ploemacher, R. E. Primary murine MSC show highly efficient homing to the bone marrow but lose homing ability following culture. Leukemia 17, 160-170 (2003).

16. Zaja-Milatovic, S. \& Richmond, A. CXC chemokines and their receptors: a case for a significant biological role in cutaneous wound healing. Histol. Histopathol. 23, 1399-1407 (2008).

17. Mei, H. et al. Chronic low-dose cadmium exposure impairs cutaneous wound healing with defective early inflammatory responses after skin injury. Toxicol. Sci. 159, 327-338 (2017).

18. Bruno, P. P., Carpino, F., Carpino, G. \& Zicari, A. An overview on immune system and migraine. Eur. Rev. Med. Pharmacol. Sci. 11, 245-248 (2007).

19. Nakamura, Y., Ishikawa, H., Kawai, K., Tabata, Y. \& Suzuki, S. Enhanced wound healing by topical administration of mesenchymal stem cells transfected with stromal cell-derived factor-1. Biomaterials 34, 9393-9400 (2013).

20. Lee, D. Y. et al. Treatment of chemically induced oral ulcer using adiposederived mesenchymal stem cell sheet. J. Oral Pathol. Med. 46, 520-527 (2017).

21. Wynn, R. F. et al. A small proportion of mesenchymal stem cells strongly expresses functionally active CXCR4 receptor capable of promoting migration to bone marrow. Blood 104, 2643-2645 (2004).

22. Iglesias-Bartolome, R. et al. mTOR inhibition prevents epithelial stem cell senescence and protects from radiation-induced mucositis. Cell Stem Cell 11, 401-414 (2012)

23. Yu, J. S. \& Cui, W. Proliferation, survival and metabolism: the role of PI3K/AKT/ mTOR signaling in pluripotency and cell fate determination. Development $\mathbf{1 4 3}$ 3050-3060 (2016).
24. Sonis, S. T. Mucositis: the impact, biology and therapeutic opportunities of oral mucositis. Oral Oncol. 45, 1015-1020 (2009).

25. Calio, M. L. et al. Transplantation of bone marrow mesenchymal stem cells decreases oxidative stress, apoptosis, and hippocampal damage in brain of a spontaneous stroke model. Free Radic. Biol. Med. 70, 141-154 (2014).

26. Chang, P. et al. Multi-therapeutic effects of human adipose-derived mesenchymal stem cells on radiation-induced intestinal injury. Cell Death Dis. 4, e685 (2013).

27. Ortiz, F. et al. Melatonin blunts the mitochondrial/NLRP3 connection and protects against radiation-induced oral mucositis. J. Pineal Res. 58, 34-49 (2015).

28. Zheng, C. et al. Prevention of radiation-induced oral mucositis after adenoviral vector-mediated transfer of the keratinocyte growth factor cDNA to mouse submandibular glands. Clin. Cancer Res. 15, 4641-4648 (2009).

29. Huang, J. et al. Genetic modification of mesenchymal stem cells over expressing CCR1 increases cell viability, migration, engraftment, and capillary density in the injured myocardium. Circ. Res. 106, 1753-1762 (2010).

30. Cheng, Z. et al. Targeted migration of mesenchymal stem cells modified with CXCR4 gene to infarcted myocardium improves cardiac performance. Mol. Ther. 16, 571-579 (2008)

31. Li, H. et al. CCR7 guides migration of mesenchymal stem cell to secondary lymphoid organs: a novel approach to separate GvHD from GvL effect. Stem Cells 32, 1890-1903 (2014).

32. McGuire, D. B. et al. Systematic review of basic oral care for the management of oral mucositis in cancer patients. Support Care Cancer 21, 3165-3177 (2013).

33. Sumikawa, S., Watanabe, S., Tanaka, M., Tanaka, A. \& Araki, H. Effect of basic fibroblast growth factor on radiation-induced oral mucositis in male Syrian hamsters. Int. J. Radiat. Biol. 93, 1-26 (2017).

34. Alvarez, E. et al. Preclinical characterization of CG53135 (FGF-20) in radiation and concomitant chemotherapy/radiation-induced oral mucositis. Clin. Cancer Res. 9, 3454-3461 (2003).

35. Goel, H. L. \& Mercurio, A. M. VEGF targets the tumour cell. Nat. Rev. Cancer 13 871-882 (2013).

36. Ellis, L. M. \& Hicklin, D. J. VEGF-targeted therapy: mechanisms of anti-tumour activity. Nat. Rev. Cancer 8, 579-591 (2008).

37. Fazio, C. \& Ricciardiello, L. Inflammation and Notch signaling: a crosstalk with opposite effects on tumorigenesis. Cell Death Dis. 7, e2515 (2016).

38. Fathke, $\mathrm{C}$. et al. Contribution of bone marrow-derived cells to skin: collagen deposition and wound repair. Stem Cells 22, 812-822 (2004).

39. Uccelli, A., Moretta, L. \& Pistoia, V. Mesenchymal stem cells in health and disease. Nat. Rev. Immunol. 8, 726-736 (2008).

40. Gnecchi, M., Zhang, Z., Ni, A. \& Dzau, V. J. Paracrine mechanisms in adult stem cell signaling and therapy. Circ. Res. 103, 1204-1219 (2008).

41. Ranganath, S. H., Levy, O., Inamdar, M. S. \& Karp, J. M. Harnessing the mesenchymal stem cell secretome for the treatment of cardiovascular disease. Cell Stem Cell 10, 244-258 (2012).

42. I., T. et al. Bone marrow-derived cell therapy for oral mucosal repair after irradiation. J. Dent. Res. 93, 813-820 (2014).

43. Cai, J. et al. ERK/Drp1-dependent mitochondrial fission is involved in the MSCinduced drug resistance of T-cell acute lymphoblastic leukemia cells. Cell Death Dis. 7, e2459 (2016)

44. Noh, H. K. et al. Electrospinning of chitin nanofibers: degradation behavior and cellular response to normal human keratinocytes and fibroblasts. Biomaterials 27, 3934-3944 (2006).

45. Leelahavanichkul, K. \& Gutkind, J. S. Oral and pharyngeal epithelial keratinocyte culture. Methods Mol. Biol. 945, 67-79 (2013). 